\title{
Research Paper: Effect of Ankle Taping and Fatigue on Dynamic Stability in Athletes With and Without Chronic Ankle Instability
}

\author{
*Tahereh Pourkhani ${ }^{1}$, Ali Asghar Norasteh ${ }^{1}$, Ali Shamsi ${ }^{1}$
}

1- Department of Sport Injuries and Corrective Exercises, Faculty of Physical Education, University of Guilan, Rasht, Iran.

$\begin{gathered}\text { Use yur devic to scan } \\ \text { and read thearticle online }\end{gathered}$
out Chronic Ankle Instability (Persian)]. Archives of Rehabilitation. 2017; 18(2):110-121. http://dx.doi.org/10.21859/jrehab-1802108
dol: $:$ http://dx.doi.org/10.21859/jrehab-1802108

Received: 31 Jan 2017 Accepted: 23 May 2017

Keywords:

taping, dynamic balance, fatigue, chronic ankle instability

\section{ABSTRACT}

Objective Lateral ankle sprains (LAS) are common among athletes and can result in a high rate of reinjury and Chronic Ankle Instability (CAI). CAI can affect dynamic stability in athletes. On the other hand, fatigue can disrupt dynamic stability in injured as well as healthy athletes. Recent studies support the use of taping and braces in subjects with chronic ankle instability. Nonetheless, it is not known if applying ankle taping can improve dynamic stability in fatigued people. So the goal of this research is to study the effect of ankle taping and fatigue on dynamic stability in female athletes with and without chronic ankle instability.

Materials \& Methods Twenty female athletes including 10 subjects with chronic ankle instability (age $22.02 \pm 1.98$ years, height $163.80 \pm 2.74 \mathrm{~cm}$, weight $58.68 \pm 7.10 \mathrm{~kg}$, FADI $80.78 \pm 1.03 \%$ and FADI Sport $65.10 \pm 1.75 \%$ ) and 10 healthy subjects (age $21.70 \pm 0.67$ years, height $162.90 \pm 5.06 \mathrm{~cm}$, weight $59.10 \pm 7.04$ kg, FADI and FADI Sport 100\%) participated in two separate testing sessions. Different conditions (no taping and with closed basket-weave ankle taping) were applied at each session. Three trials of a jump landing task were performed under each condition before and after induced functional fatigue. The jump-landing task involved a single-leg landing onto a force plate from a height equivalent to 50 percent of each participant's maximal jump height and from a starting position $70 \mathrm{~cm}$ from the center of the force plate. The functional fatigue protocol comprised three stations: Modified Southeast Missouri (SEMO) agility drill, stationary lunges, and quick jumps. The participants continued to run through each station until the time to finish the stations increased by $50 \%$ compared with their baseline timed runs. Time to stabilization was measured in the anterior-posterior (APTTS), medial-lateral (MLTTS) and vertical directions. Three separate repeated-measure analyses of variance with two within-subjects factor (condition and time) were performed for each dependent variable in each group..

Results The results of this investigation revealed that in healthy athletes group, the difference between MLTTS and vertical TTS was statistically significant $\{f=7.52, P=0.001\},\{f=9.69, P=0.004\}$. Bonferroni post hoc testing revealed faster pretest MLTTS than posttest for taping condition and faster pretest vertical TTS than posttest for no taping condition. In injured athletes group, the difference between MLTTS and vertical TTS was statistically significant $\{\mathrm{f}=10.57, \mathrm{P}=0.001\},\{\mathrm{f}=14.27, \mathrm{P}=0.001\}$. This testing also revealed faster pretest MLTTS than posttest for no taping condition, faster pretest vertical TTS than posttest for both conditions, and faster vertical TTS after taping than before taping.

Conclusion In the athletes with chronic ankle instability, taping without fatigue improved dynamic balance in the vertical direction. Taping after fatigue could not improve dynamic stability in the athletes with and without chronic ankle instability. Future researchers should examine injured and uninjured participants tested under these conditions to determine if these results are useful in selecting appropriate prophylactic method that can treat or prevent injury to the ankle during functional activities.

\section{* Corresponding Author:}

Tahereh Pourkhani, PhD Student

Address: Department of Sport Injuries and Corrective Exercises, Faculty of Physical Education, University of Guilan, Rasht, Iran.

Tel: +98 (911) 3410729

E-Mail: zpourkhani@gmail.com 


\title{
اثر نواربندى و خستكى بر ثبات يويا در ورزشكاران باو بدون بىثباتى مزمن مج ها
}

\author{
•طاهره يورخانى'، على اصغر نورسته'، على شمسى' \\ 1- كروه آسيبشناسى ورزشى و حركات اصلاحى، دانشكده تربيت بلنى و علوم ورزشى، دانشعاه كيلان، رشت، ايران.
}

\begin{abstract}
حكبن

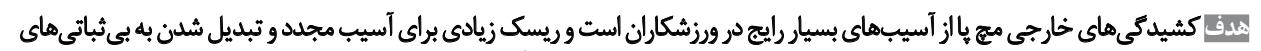

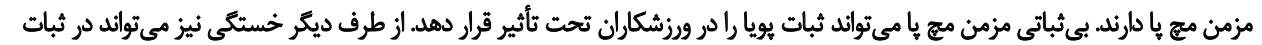

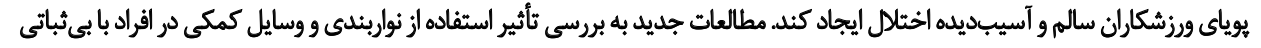

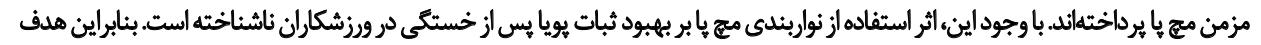

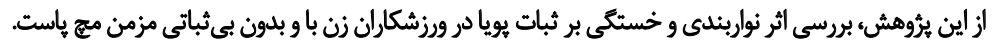

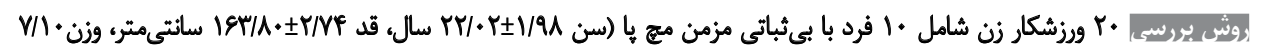

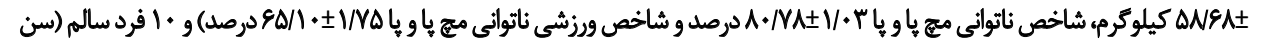

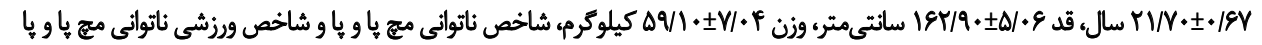

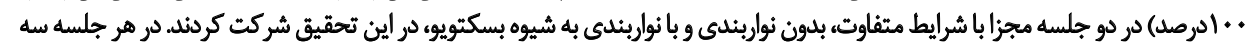

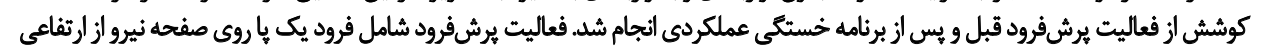

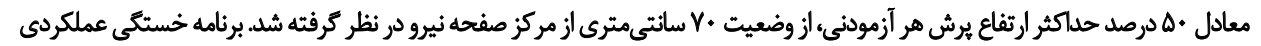

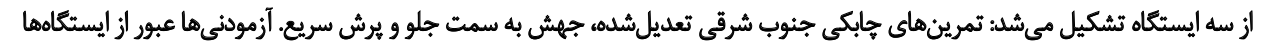

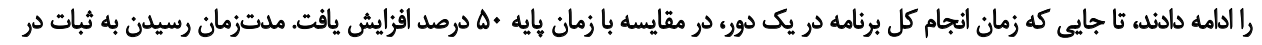

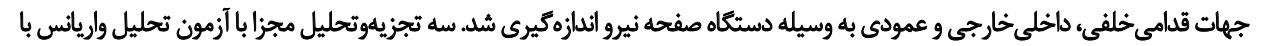

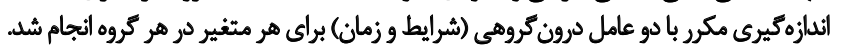

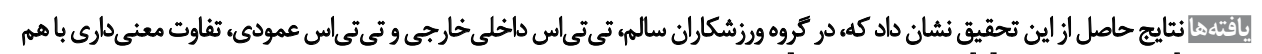

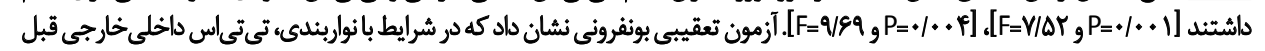

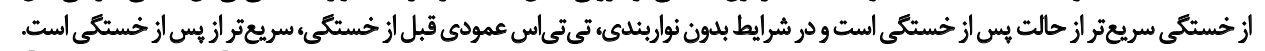

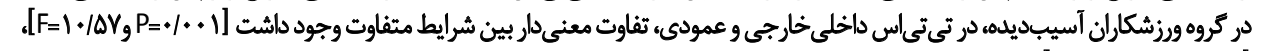

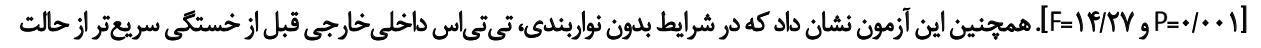

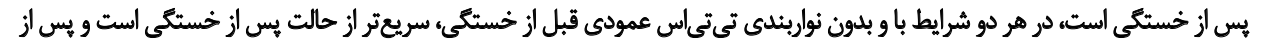
نواربندى سريعتر ازقبل از نواربندى است.

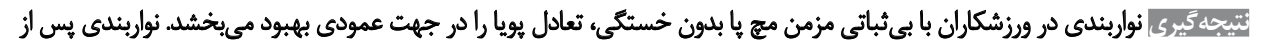

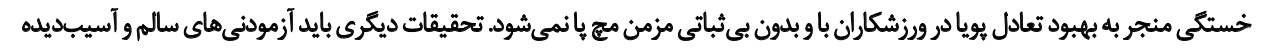

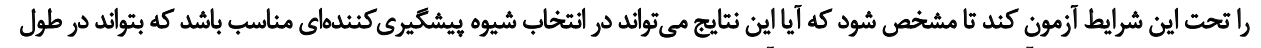

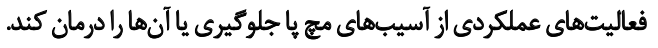

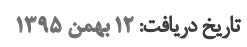

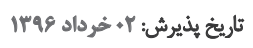

كليدوأوهاء:

نواربندى، تعادل يويا،

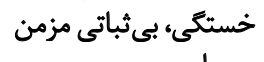

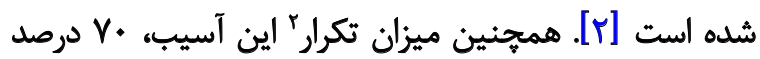

مقدمه

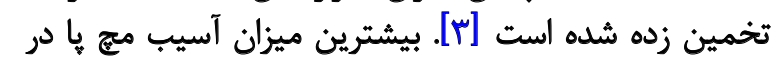

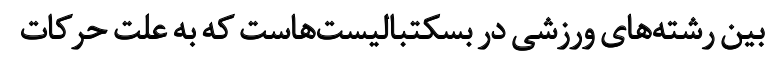
يرشى ناتكهانى در حين فرار از مدافع و فرودهاى نامتعادل حين بن بركات

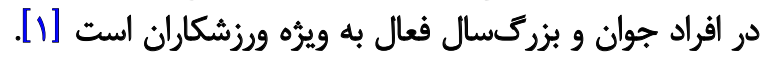

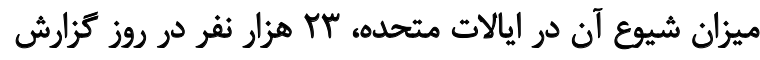

1. Lateral Ankle Sprain (LAS)

\section{* نويسئده مسئول: \\ طاهره هورخ نوخاني} نشانى: رشت، دانشكاه كيلان، دانشكده تربيت بلنى و علوم ورزشى، كروه آسيبشناسى ورزشى و حركات اصلاحى.

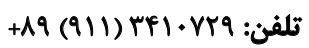
رايانامه: zpourkhani@gmail.com 


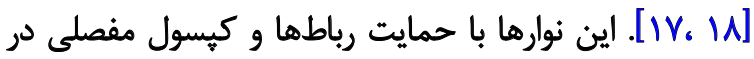

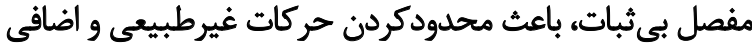

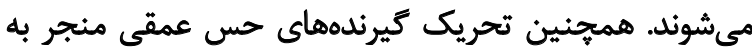

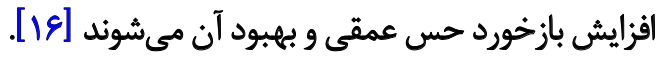

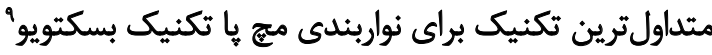

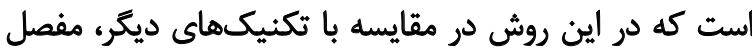

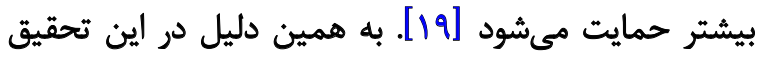

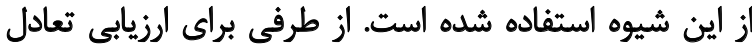

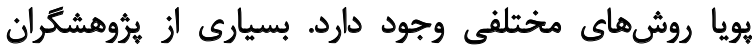

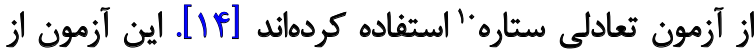

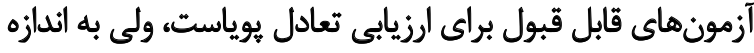

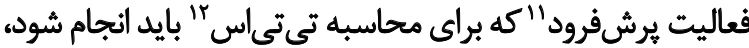

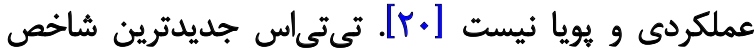

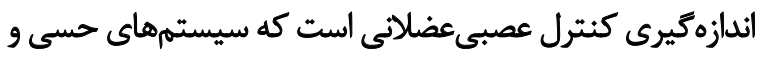

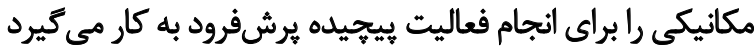

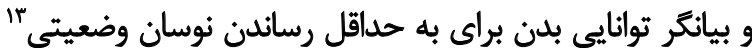

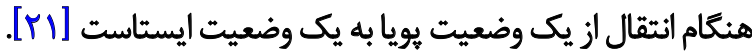

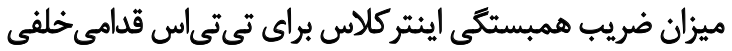

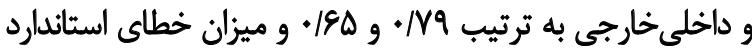

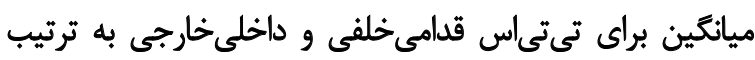

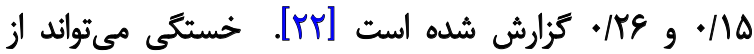

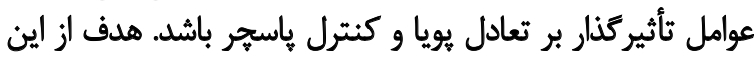

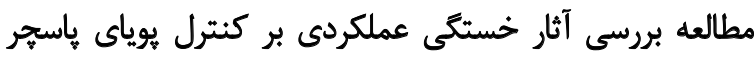

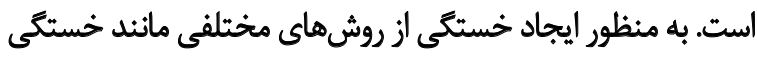

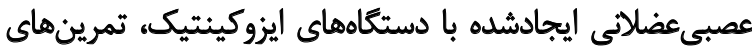

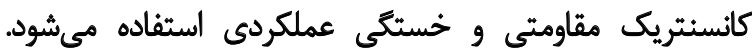

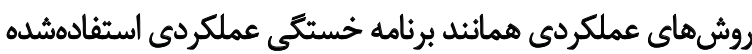

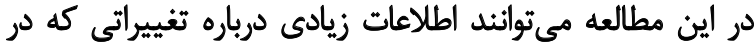

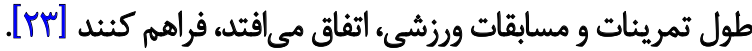

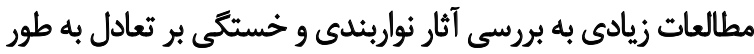

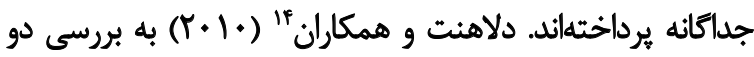

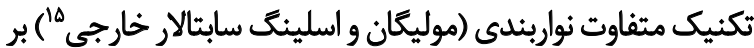

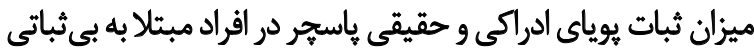

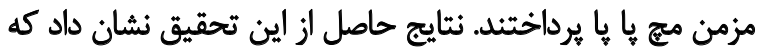

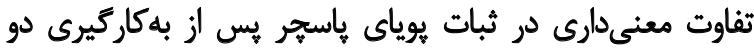

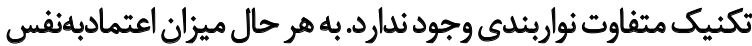
و ثبات و اطمينان در آزمودنىها يس از استفاده از نواربندى افزايش

9. Basketweave Technique

10. Star Excursion Balance Test

11. Jump Landing Task

12. Time to stabilization

13. Postural sway

14. Delahunt et al.

15. Lateral subtalar sling

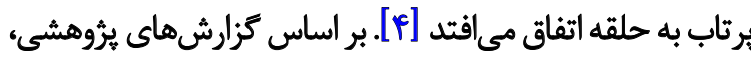

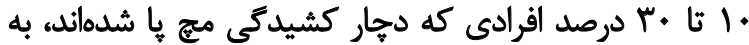

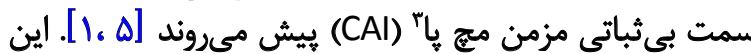

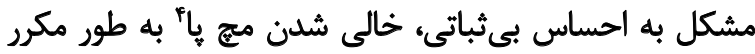

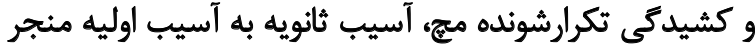

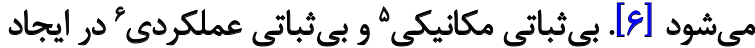

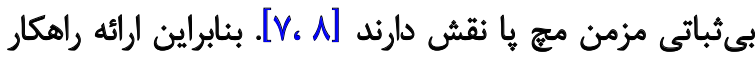

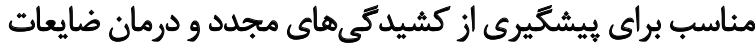

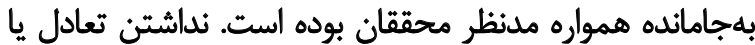

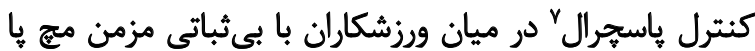

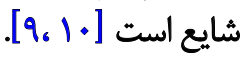

حفظ تعادل در يك زنجيره بسته كينتيكى انجام مىشودي،

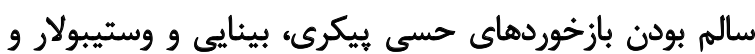

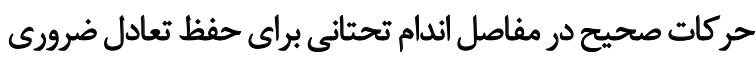

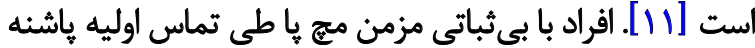

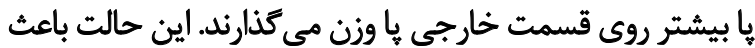

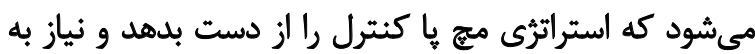

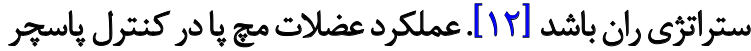

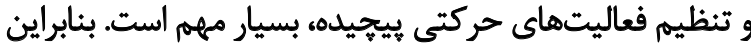

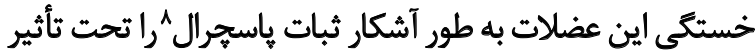

قرار مى دهد إن [1]]

حستكى به دو نوع مركزى و محيطى تقسيم مي بشود. كاهش

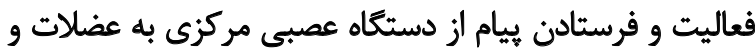
ناتوانى براي توليد تنش مناسي

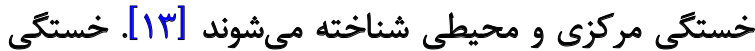

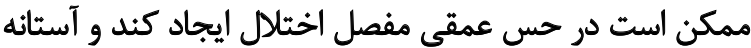

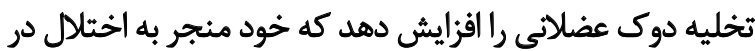

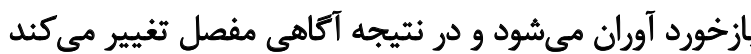

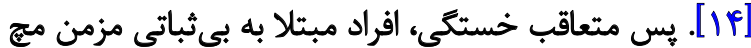

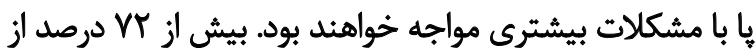

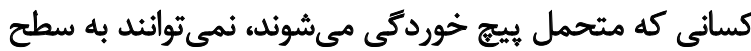

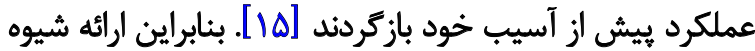

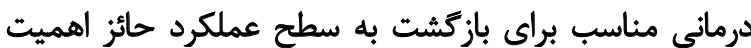

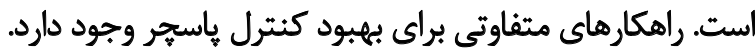

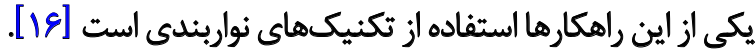

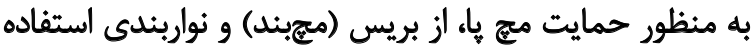

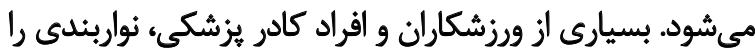

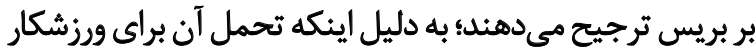
راحتثر است و در عملكرد طبيعى مفصل اختلال ايجاد نمى كند تهند

\footnotetext{
3. Chronic Ankle Instability (CAI)

4. Giving way

5. Mechanical instability

6. Functional instability

7. Postural control

8. Postural stability
} 


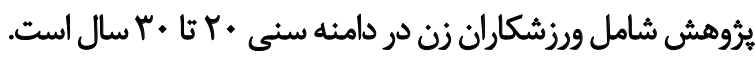

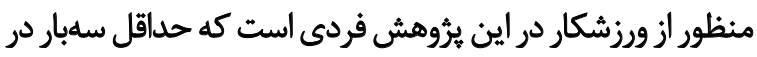

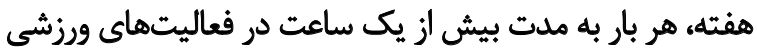

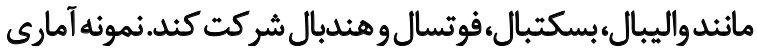

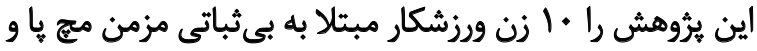

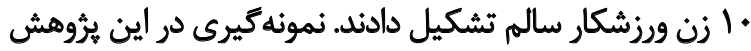

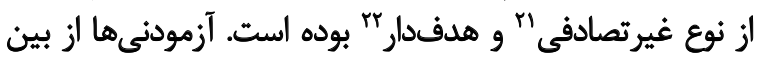

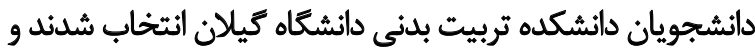

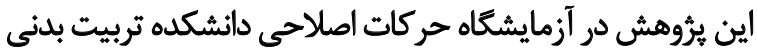

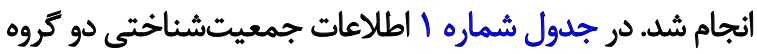

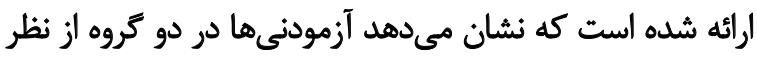
سن، قد، وزن و طول اندام تحتانى با هم تففاوتى ندارند.

ابتدا افراد با سابقه كشيدكى خارجى منج يا از طريق شناسايى

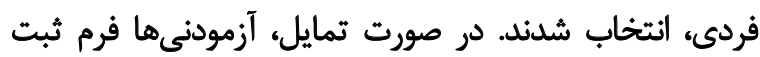

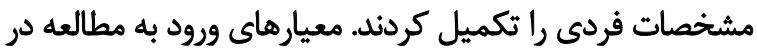

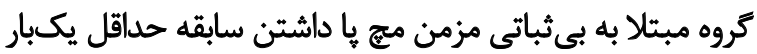

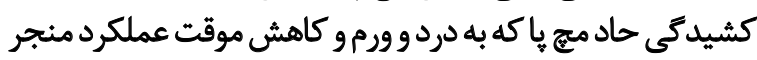

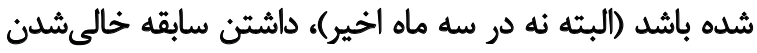

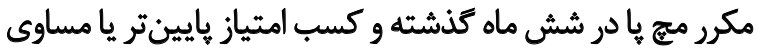

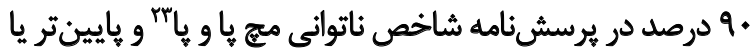

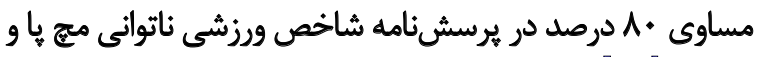

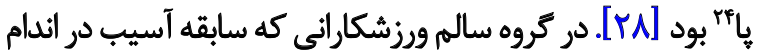

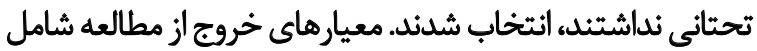

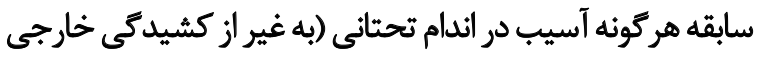

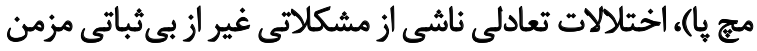

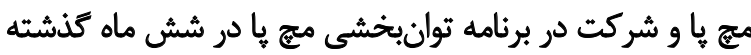

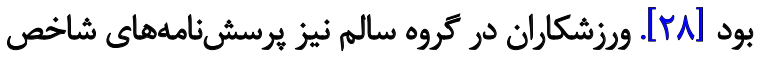

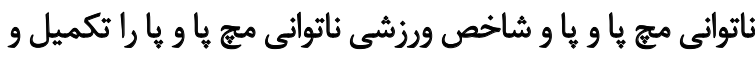
در صورت كسب امتياز صددرصد در تحقيق شركت كردند. قبل از انجام يُروهش تمام آزمودنىها، رضايتنامه آكاهانه ران

21. Non-probability sampling

22. Purposive

23. Foot Ankle Disability Index

24. Foot Ankle Disability Index Sport

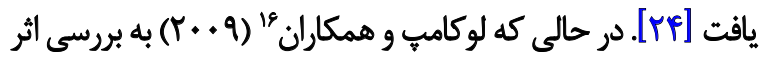

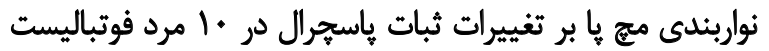

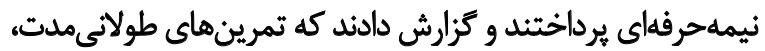

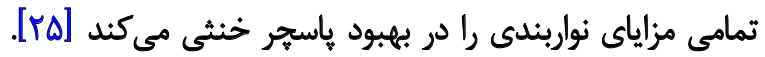

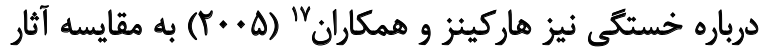

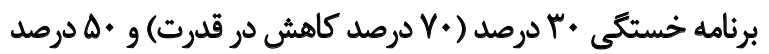

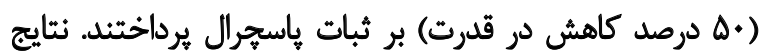

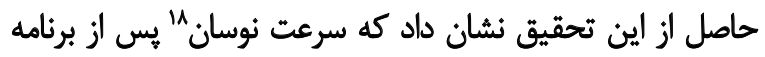

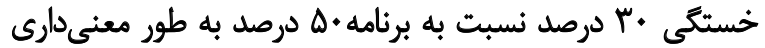

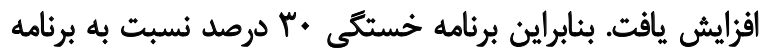

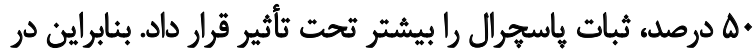

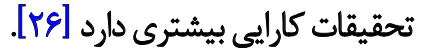

بيسون و همكاران 19 (11 + (T) به ارزيابي آثار خستخى عضلات

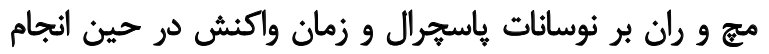

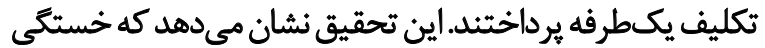

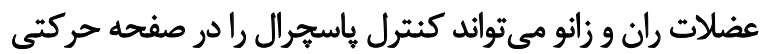

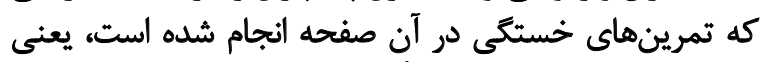

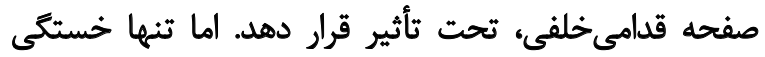

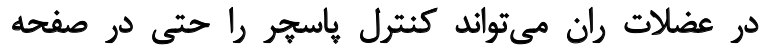

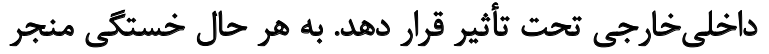

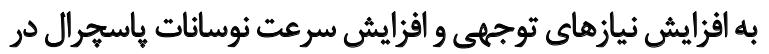

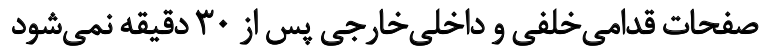

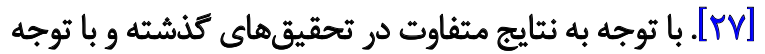

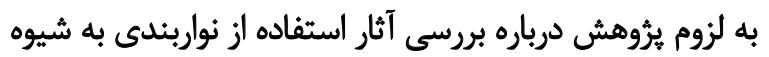

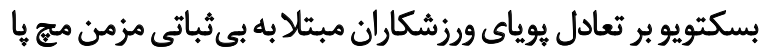
يس از خستكى، اين تحقيق انجام شد. ورشّ بروسي اين ثرُوهش ماهيت نيمهتجربى · دارد. جامعه آمارى اين

16. Lohkamp et al.

17. Harkins et al.

18. Sway velocity

19. Bisson et al.

20. Quasi-experimental

جدول ا ـ اطلاعات نموكر افيك آزمودنىها

\begin{tabular}{|c|c|c|c|c|}
\hline $\mathbf{T}$ & Sig. & MeantS.D & $\begin{array}{l}\text { تحروه با بي ثباتى مزمن ميج ها } \\
\text { MeantS.D }\end{array}$ & \\
\hline . NAT &.$M I r$ & $r W N \pm \pm . / Q V$ & $r r / r \cdot \pm 1 / u$ & سن (سال) \\
\hline . /FAT &.$N \cdot 1$ & $\mid q T / q \cdot \pm \Delta / 98$ & $19 / / \lambda \cdot \pm T / M^{2}$ & قد (سانتىمثر) \\
\hline.$- / 1 \cdot 1$ &.$/ 998$ & $\Delta Q / 1+ \pm V /+4$ & $\triangle N$ FN $\pm V / \Lambda$. & وزن (كيلوكرم) \\
\hline.$/ \Delta q \Delta$ & . NEA & $A r / q \cdot \pm r / \Delta 1$ & $1 F / \Delta \cdot \pm 1 / 9 \Delta$ & طول اثدام تحثانى (ساتنى متر) \\
\hline
\end{tabular}




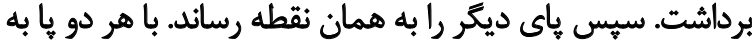

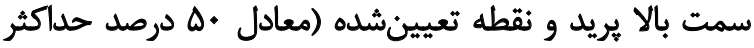

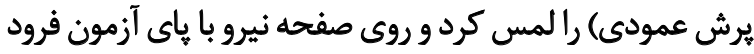

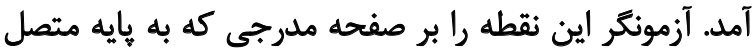

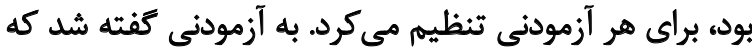

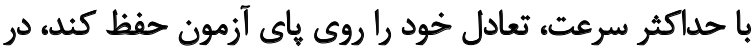

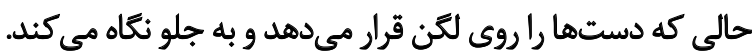

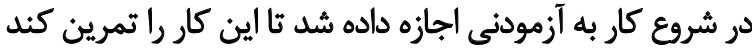

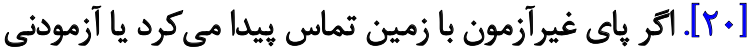
حركت اضافى انجام مى داد، آزمون تكرار مي نيد.

$$
\text { برنامه خُستكّي عملكردى }
$$

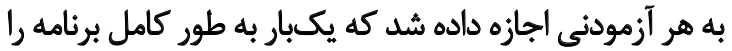

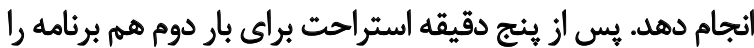

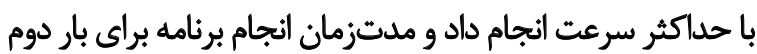

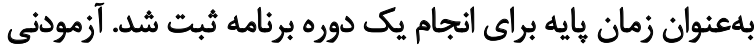

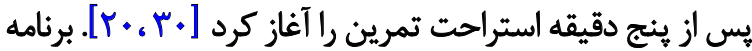

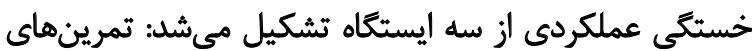

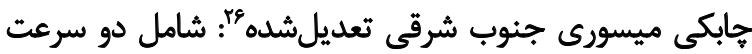

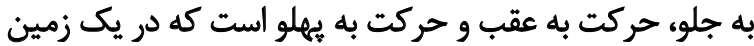

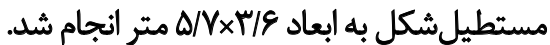

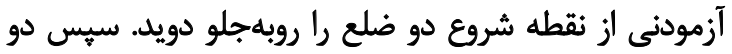

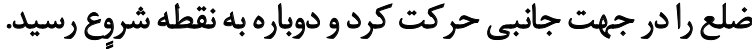

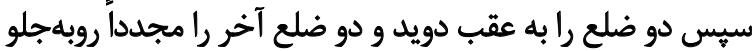

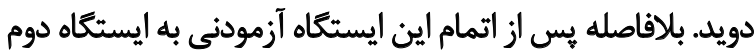

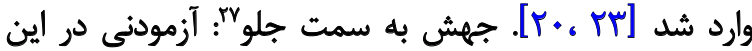

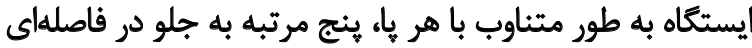

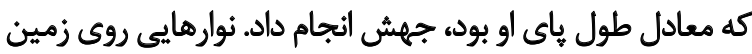

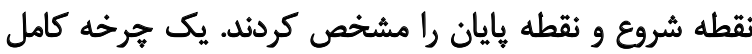

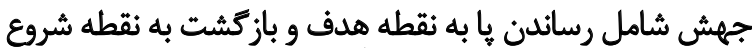

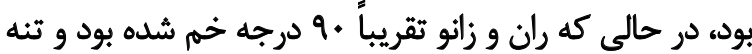

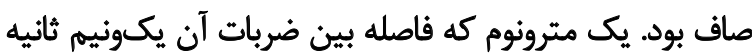

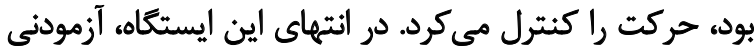

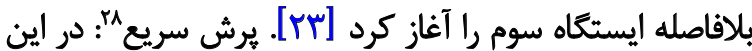

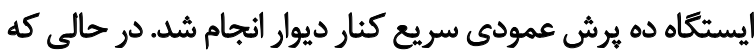

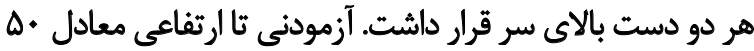

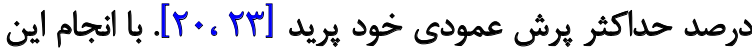

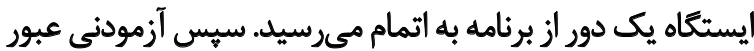

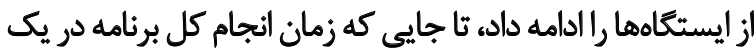

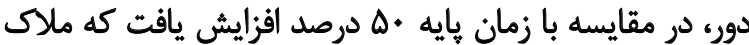

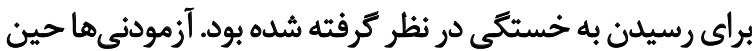

26. Modified Southeast Missouri Agility Drill

27. Stationary lunge

28. Quick jump
تكميل كردند. در اين بيروهشه اطلاعات از آزمودنىها طى دو

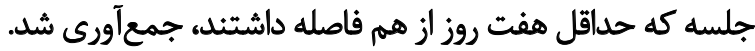

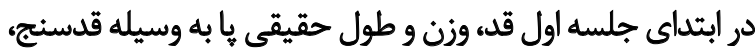

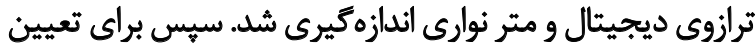

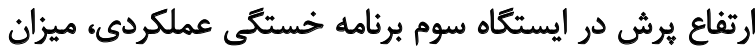

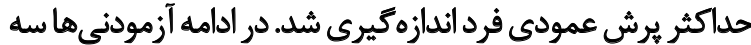

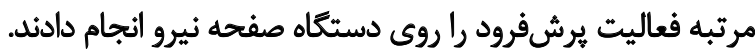

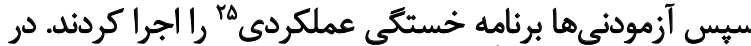

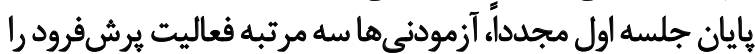

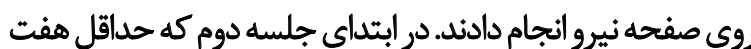

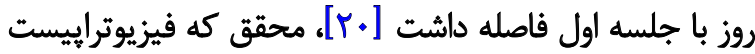

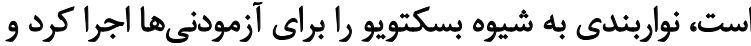

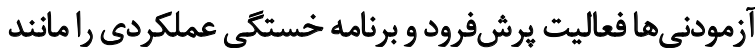

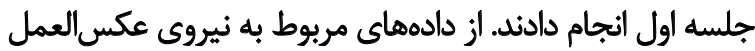

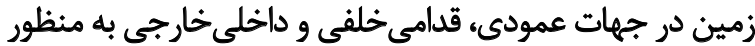

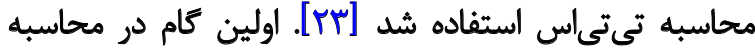

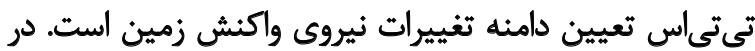

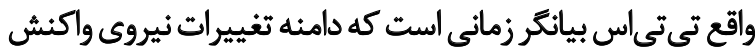

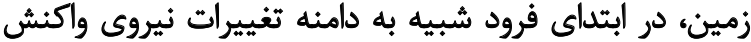

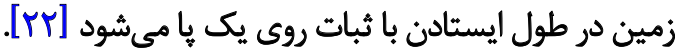

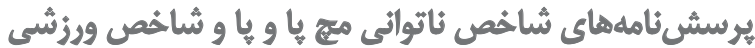

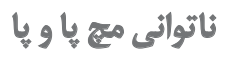

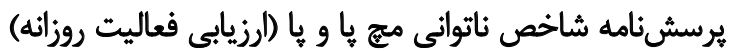

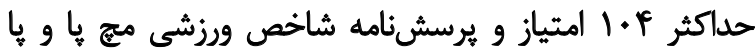

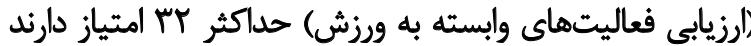

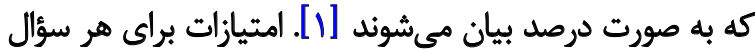

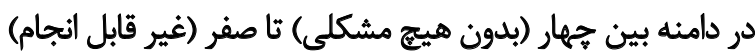

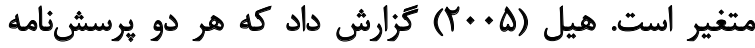

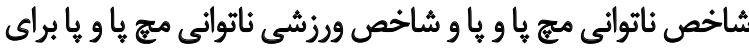

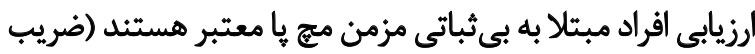

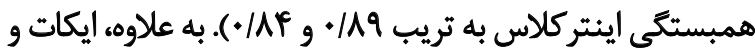

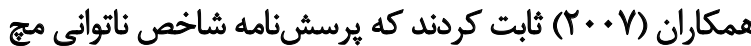

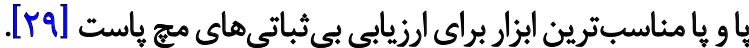
فعاليت هيرش فرود روى صفحه نيرو براى ارزيابى فعاليت يرشفرود از دستًاه صفحه نيرو (ساخت

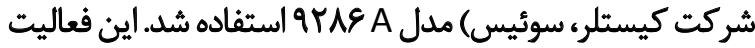

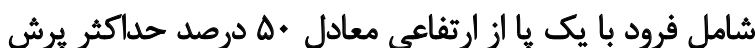

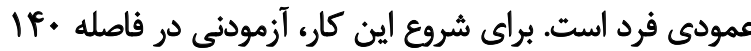

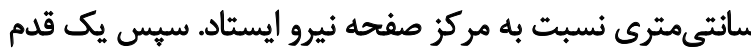

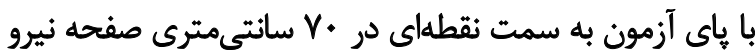

25. Functional Fatigue Protoco 
منظور تفسير دادهها از آمار استنباطى استفاده شد. براي ارزيابى إني

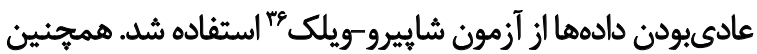

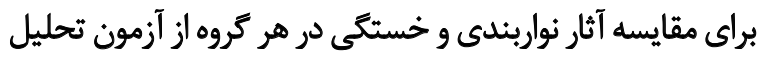

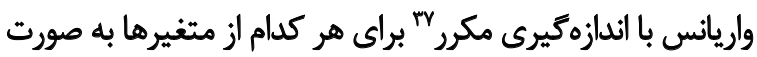

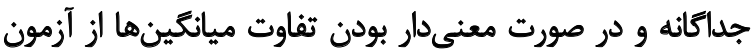

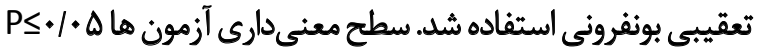

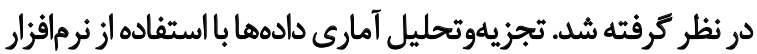

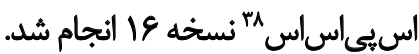

ladiós

ضريب همبستكى اينتركلاس محاسبه شده توسط يرؤوهشكر

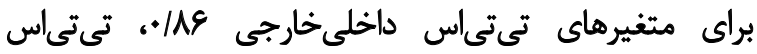

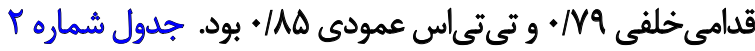

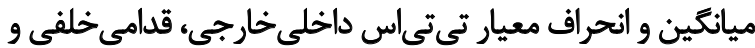
عمودى را در دو كروه نشان مي دهيد.

نتايج حاصل از اين تحقيق نشان داد كه در كروه ورزشكاران

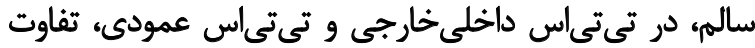

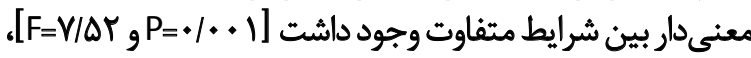

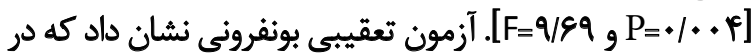

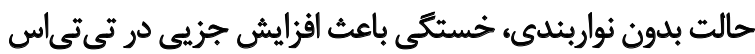

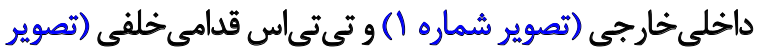

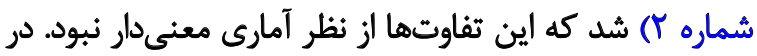

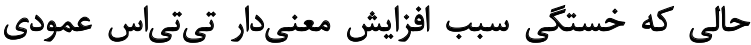

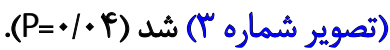

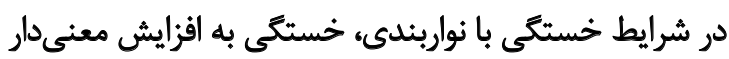

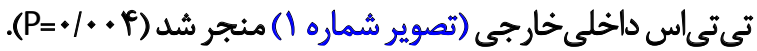

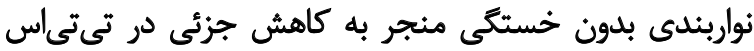

36. Shapiro-wilk

37. Repeated measures analyses of variance 38. SPSS

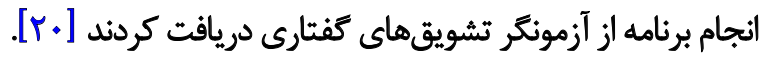

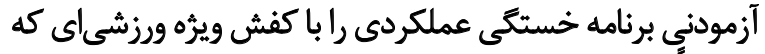
معمولاً ميى يوشيد، انجام دادي

$$
\text { ثواربثدى به شيوه بسكتويو }
$$

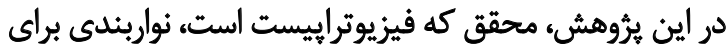

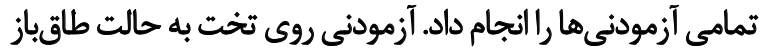

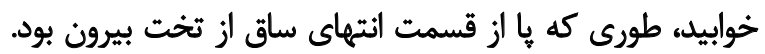

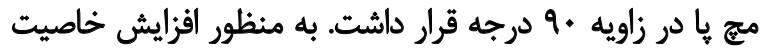

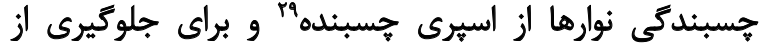

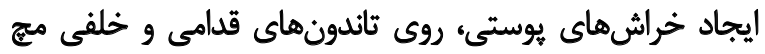

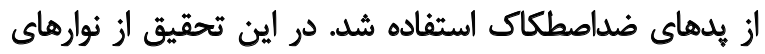

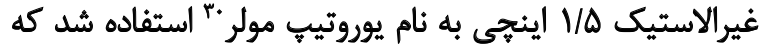

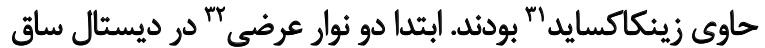

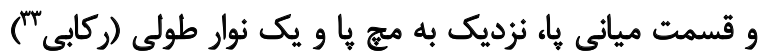

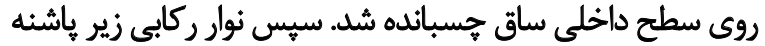

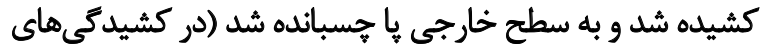

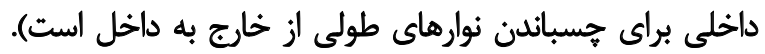

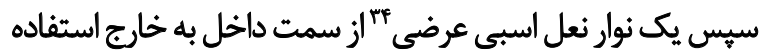

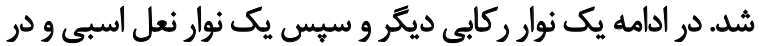

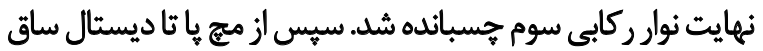

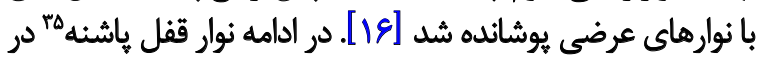

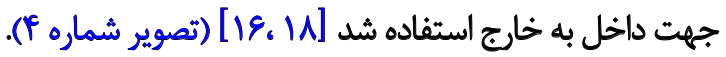
در تحقيق حاضر، به منظور سازمانبندي، خلاصه كردن ومداسبه ميانكين و انحراف استاندارد اطلاعات كمى، از آمار توصيفى و به به

29. Adhesive spray

30. Euro tape Muller

31. Zinc oxid

32. Anchor strip

33. Stirrup strip

34. Horizontal horseshoe strip

35. Heel lock

جدول r. ميانكين و انحراف استاندارد تى تىىاس در حالات متفاوت در دو كروه

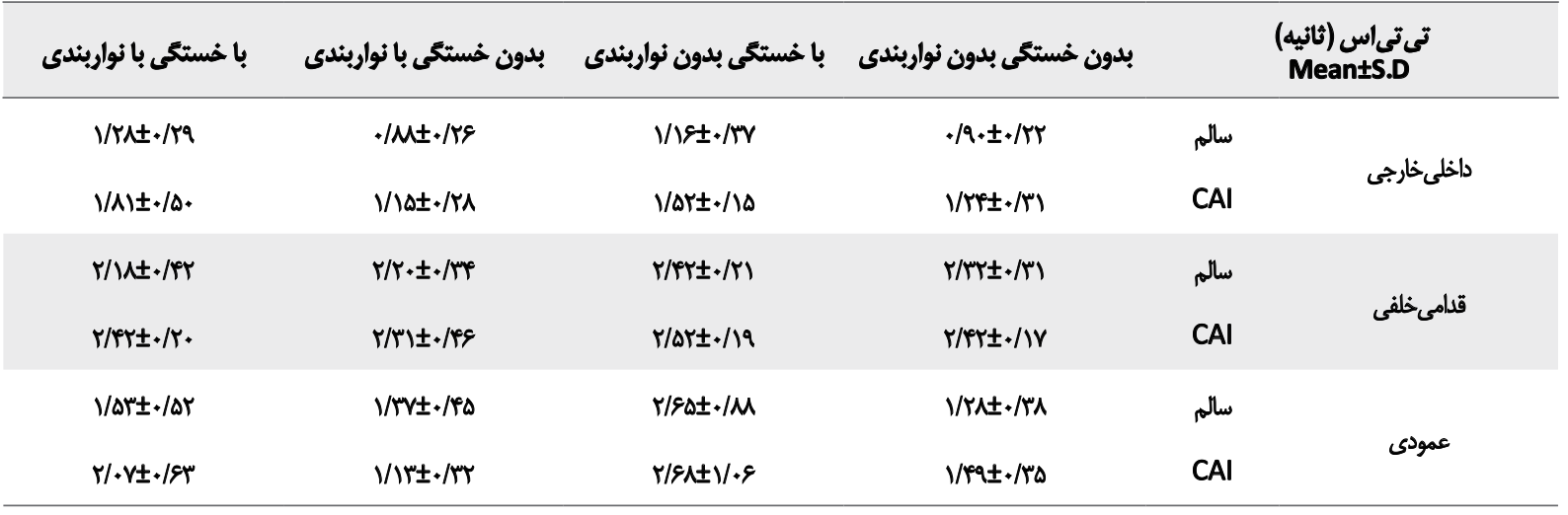



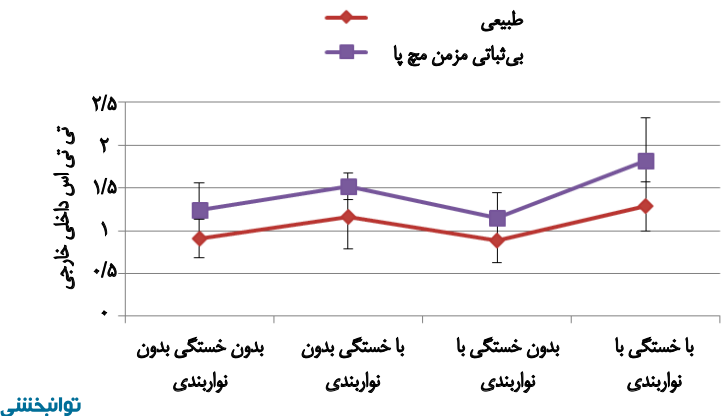

تصوير r. ميزان تغييرات تى تىاس قدامى خلفى در دو كروه در حالات متفاوت

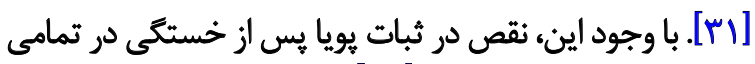

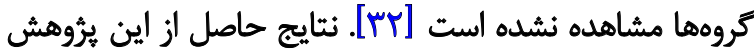

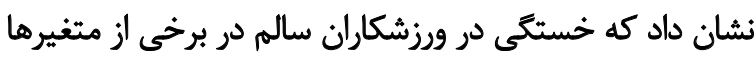

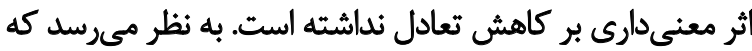

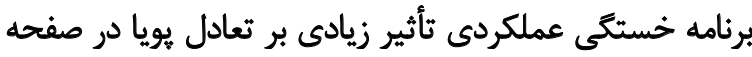

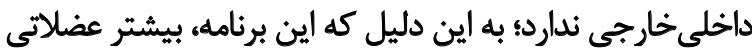

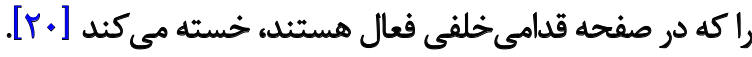

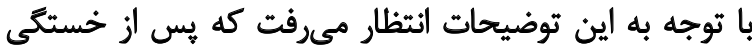

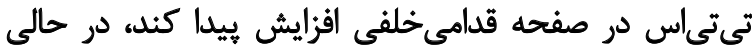

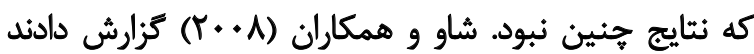

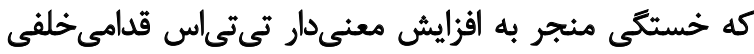

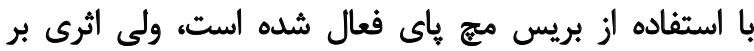

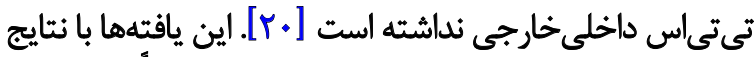

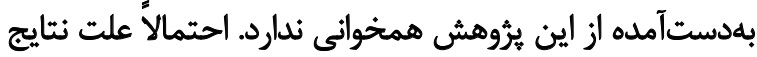

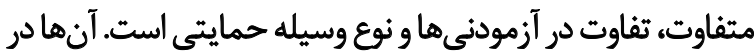

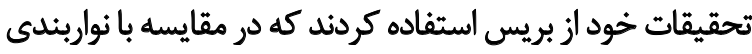

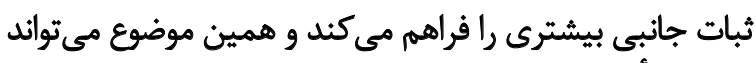

$$
\text { بر بتايج تأثير بكذارد. }
$$

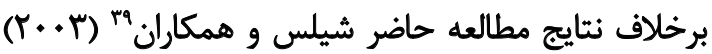

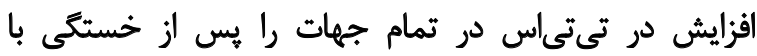

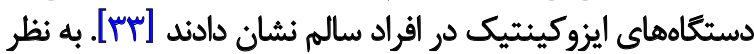

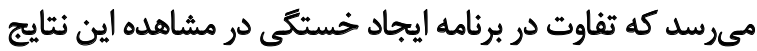

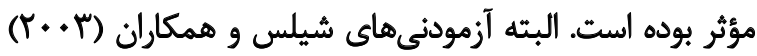

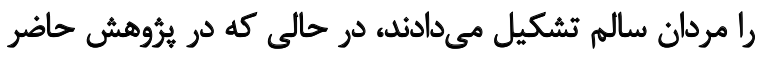

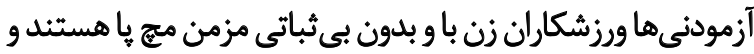

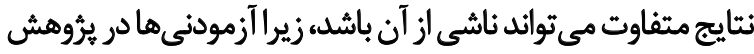

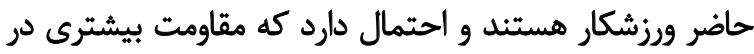

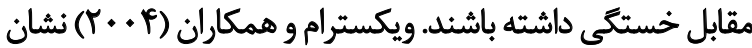

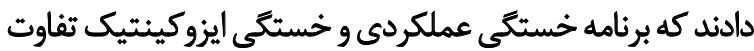

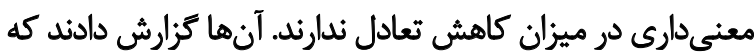

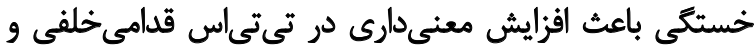

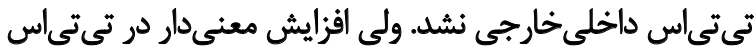

39. Shills et al.

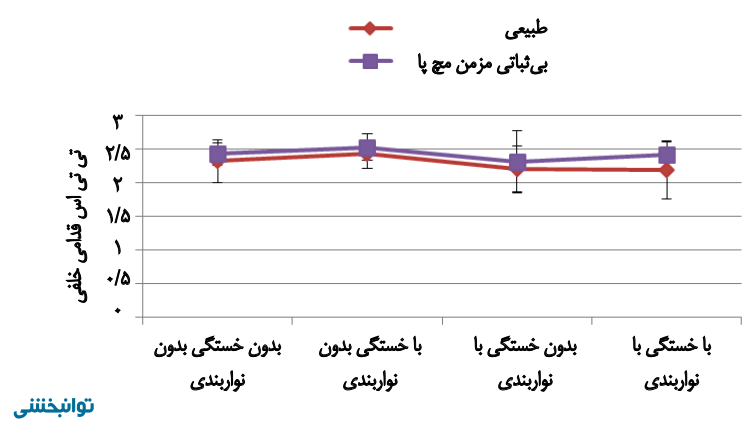

تصوير ا. ميزان تغييرات تى تىاس داخلى خارجى در دو كروه در حالات متفاوت

داخلى خارجى (تصوير شماره () و قدامى خلفى (تصوير شماره r)

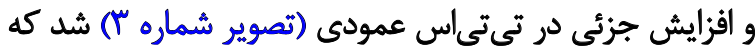

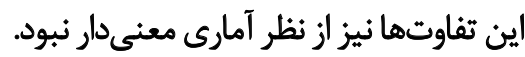

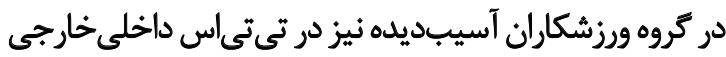

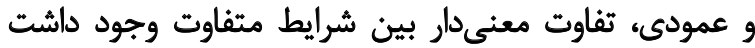

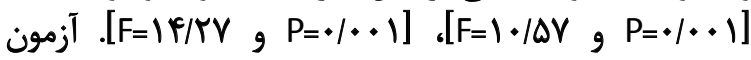

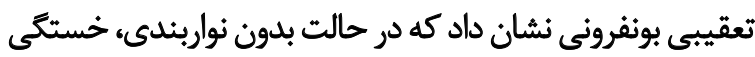

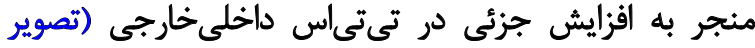

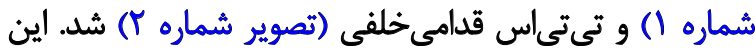

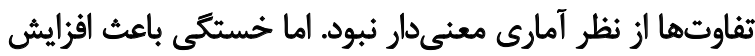

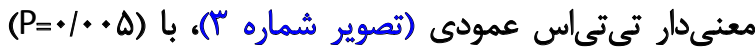

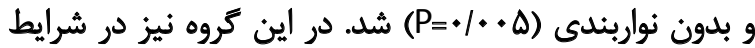

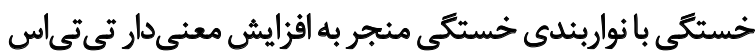

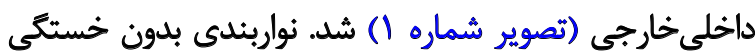

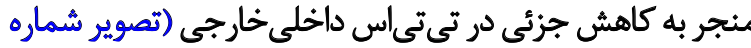

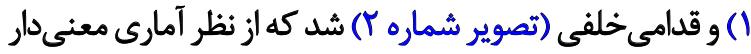

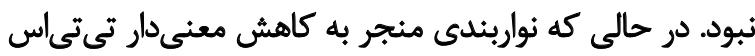

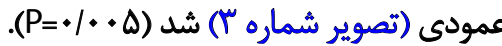

$\stackrel{\leftrightarrow}{\leftrightarrow}$

كنترل عصبى عضلانى نقش بسيار مهمي را در ثبات يوياي إني

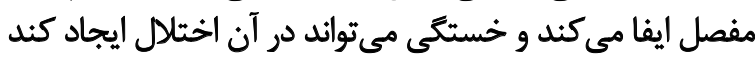

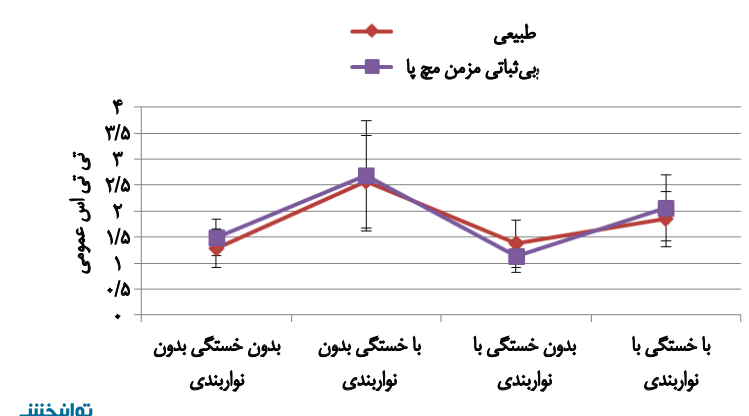

توانبخننى

تصوير ب. ميزان تغييرات تى تيىاس عمودى در دو كروه در حالات متفاوت 

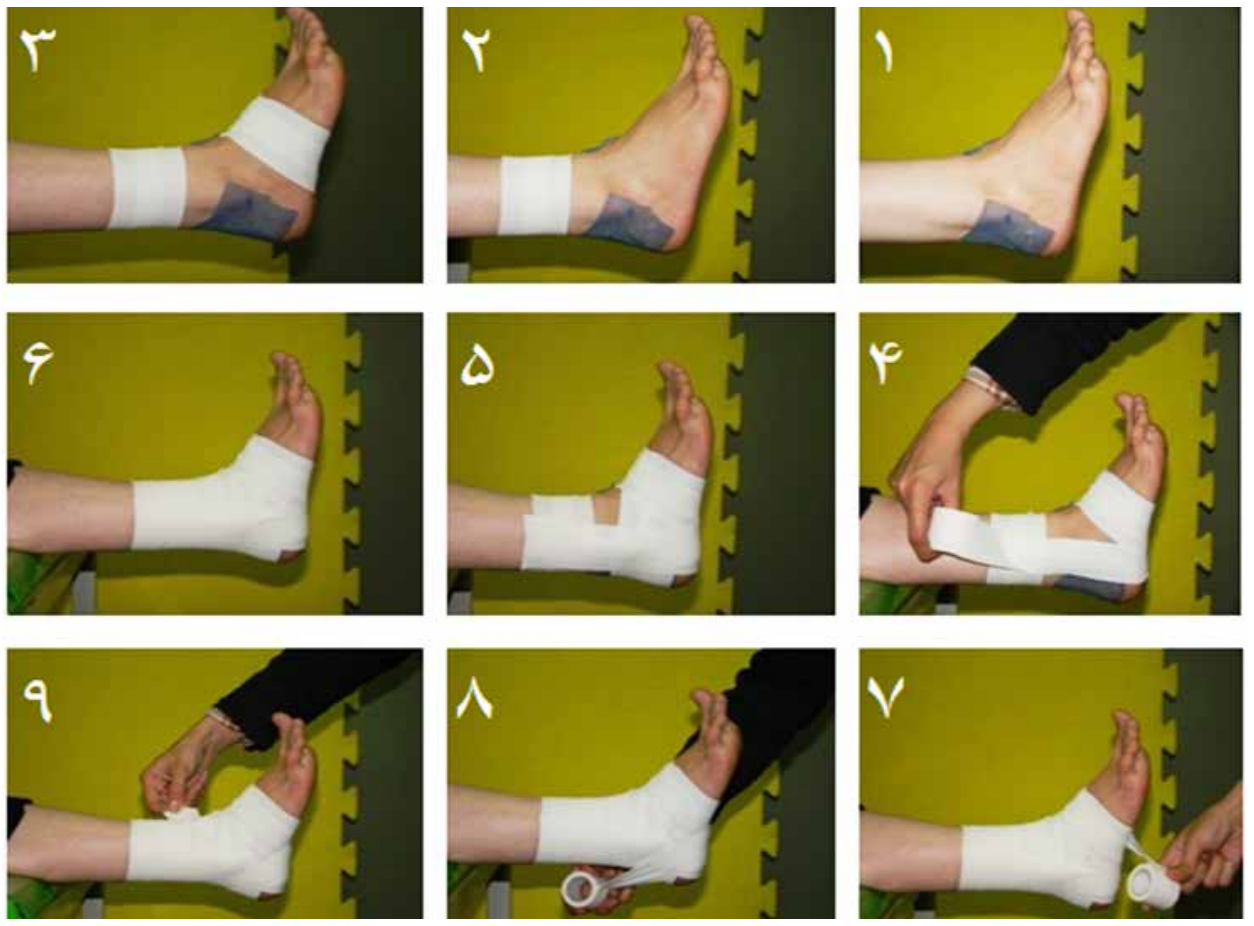

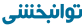

تصوير f. نحوه انجام نواربندى به شيوه بسكت ويو

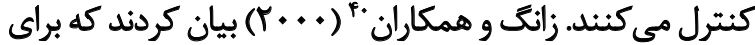

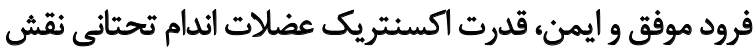

حياتى ايفا مى كند [rهis].

در لحظه فروده ابتدا عضلات مجز و سيس زانو و در نهايت

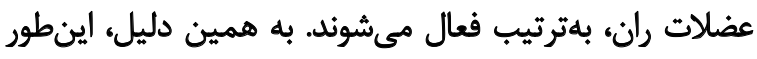

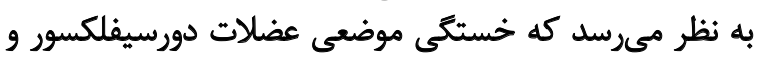

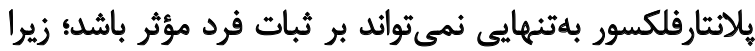

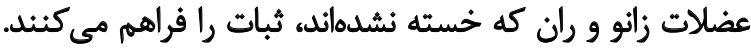

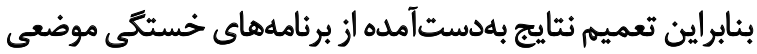

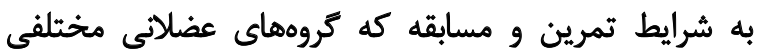

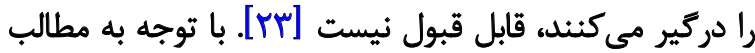

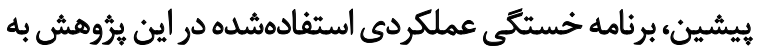

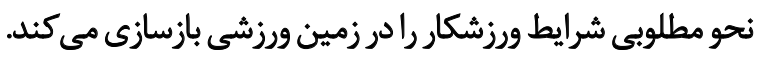

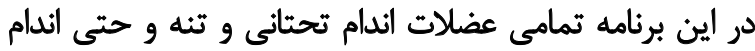

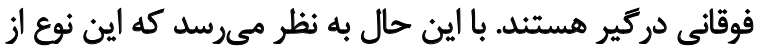

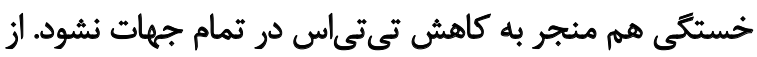

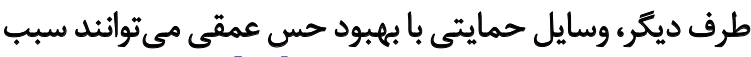
بهبود كنترل پاسجر و تعادل فرد شوند [عبائ.

در يروهش حاضر، به منظور حمايت مفصل از نواربندى استفاده

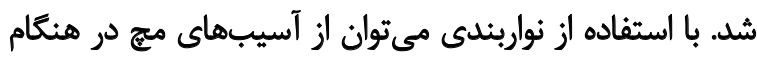

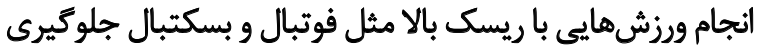

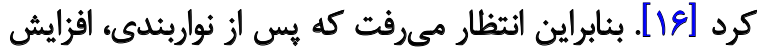

عمودى مشاهده شد [rr] در برنامه خستخى ايزوكينتيك، ملاك

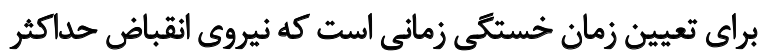

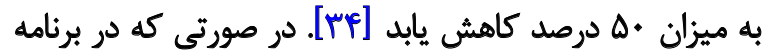

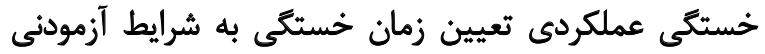

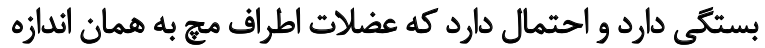

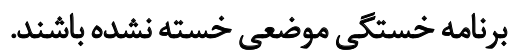
البته برخى از نتايج حاصل از يثروش ويكسترام و همكاران

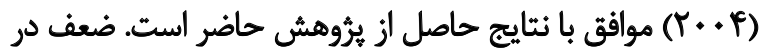

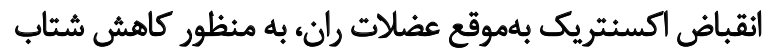

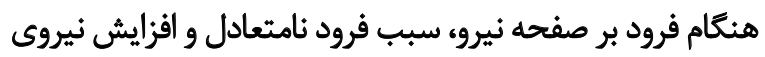

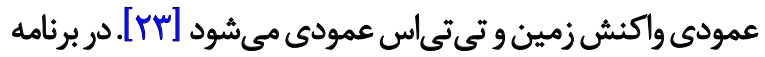

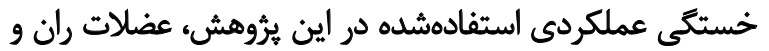

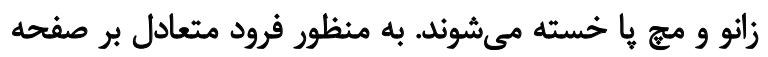

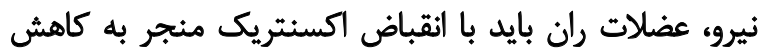

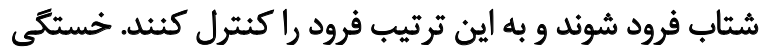

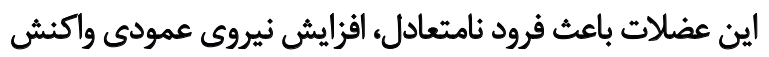

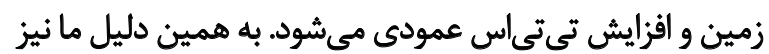

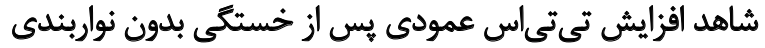

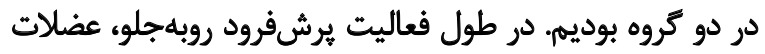

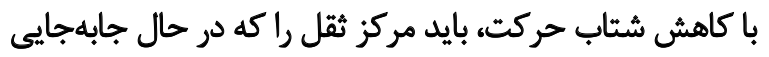

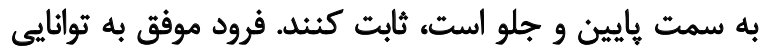
بلدن براى كاهش سرعت روبهائايين اندام تحتانى وابسته است

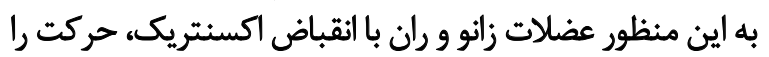

40. Zhang et al. 


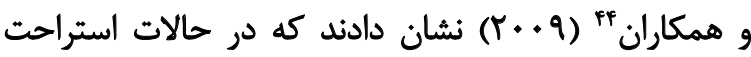

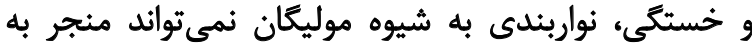

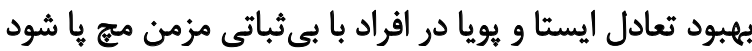

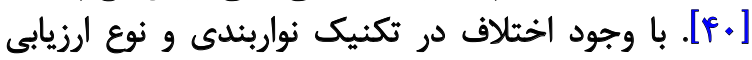

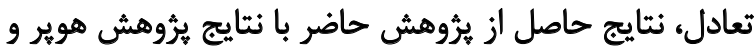

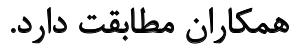

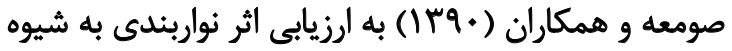

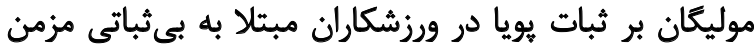

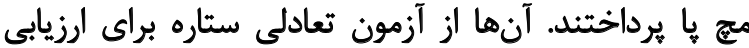

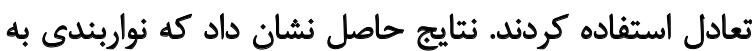

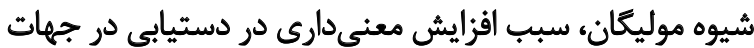

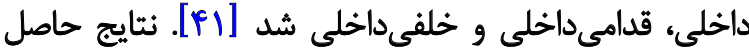

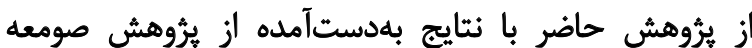

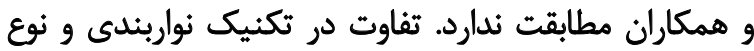

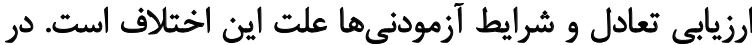

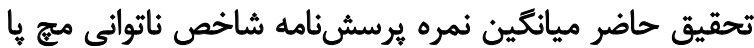

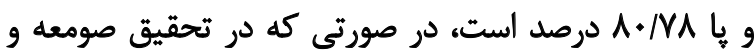

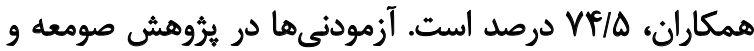

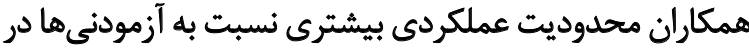

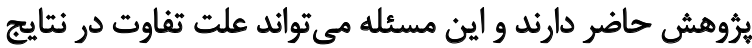

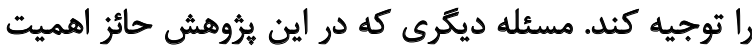

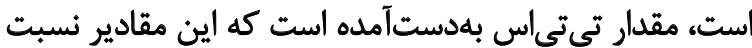
به مقادير كزارش شده در تحقيقات كذشتثه كمتر است.

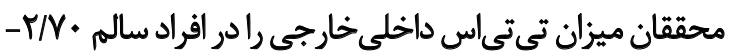
تي

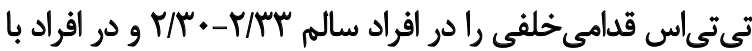

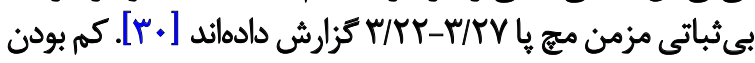

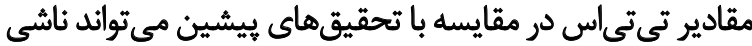

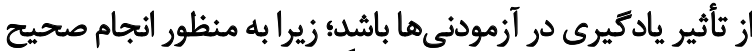

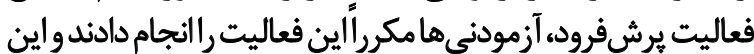

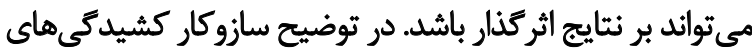

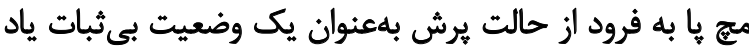

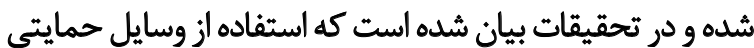

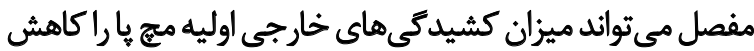

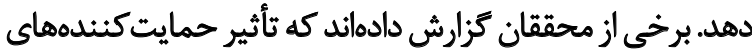

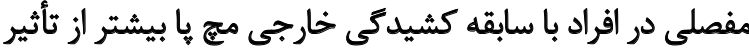

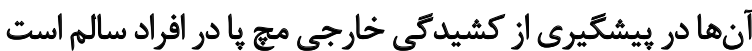

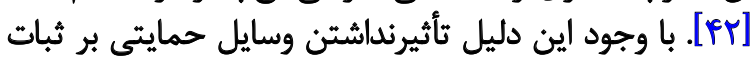

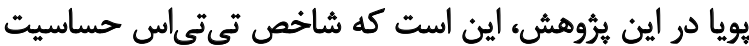

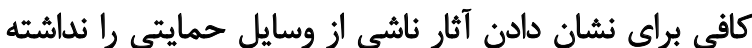

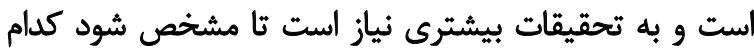
آزمون عملكردى قادر است آثار استفاده از وسايل حمايتي ماتخد ماندي

44. Hoper et al.
معنى داري در بهبود تعادل در دو گروه داشته باشيم. در صورتى

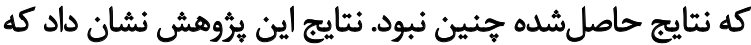

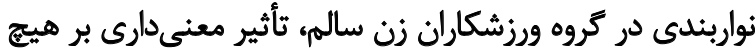

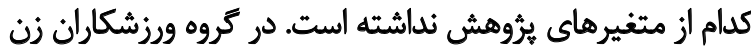

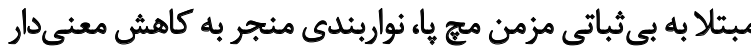

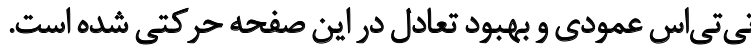

كريبل و همكاران (· (T) براي ارزيابى تعادل يويا از متغيرى

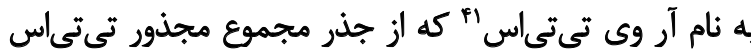

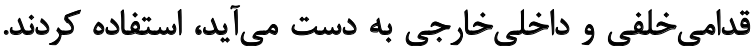

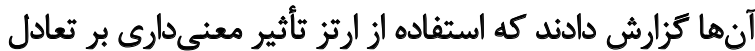

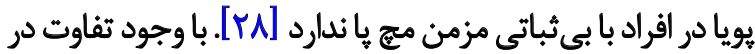

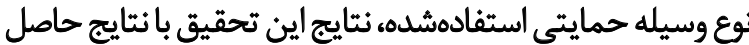

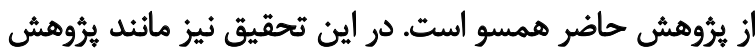

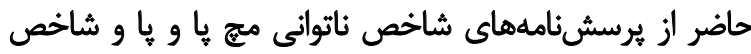

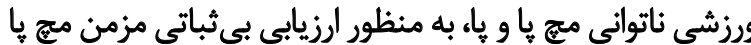

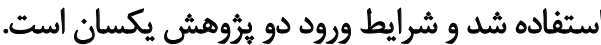

آر وى تى تىىاس متغيرى است كه با استفاده از تىتى ماس

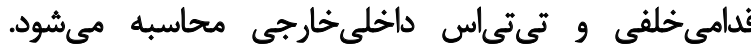

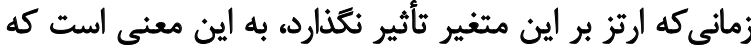

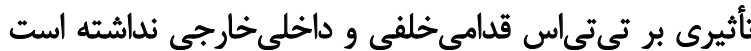

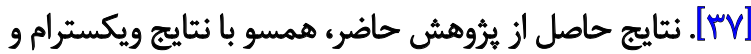

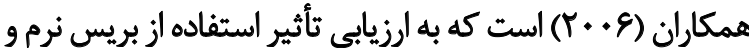

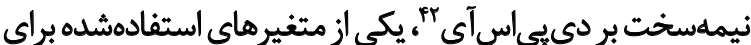

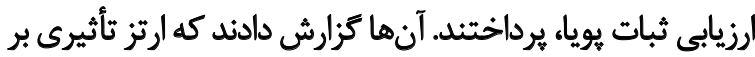

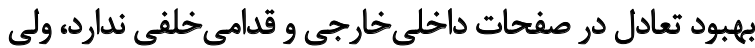

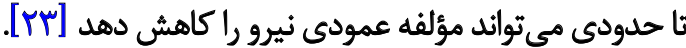

تى تىىاس براى مشخص كردن اختلالات ثبات باسجرال

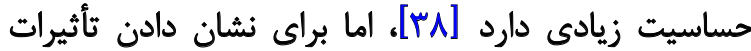

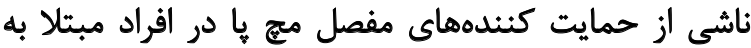

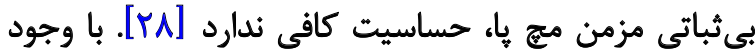

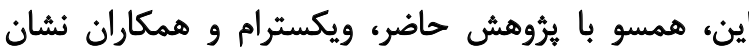

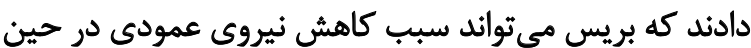

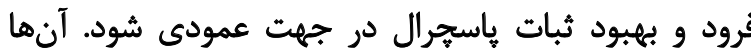

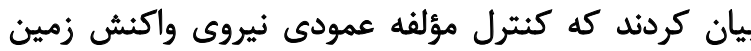

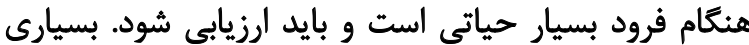

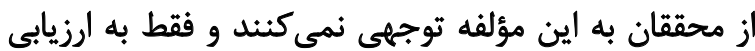

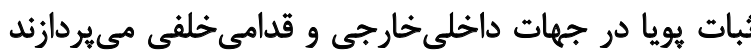

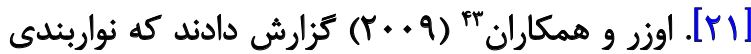

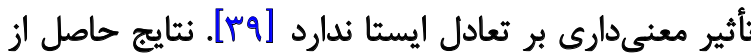

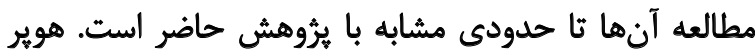

41. Resultant Vector of TTS

42. Dynamic Postural Stability Index

43. Ozer et al. 


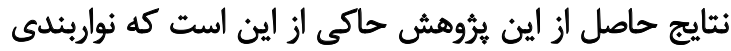

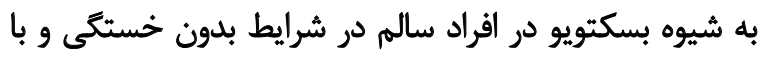

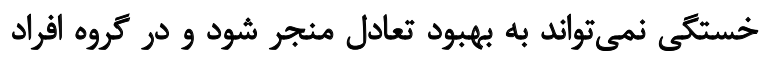

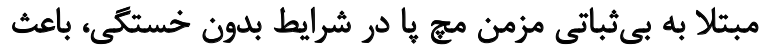

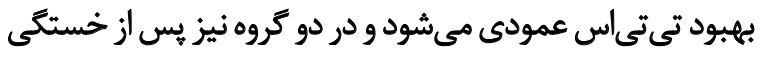

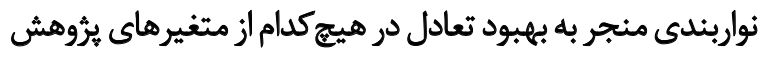

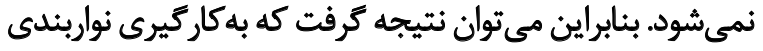

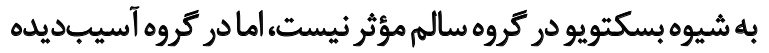
مى متواند تا حدودى به بهبود تعادل كمك كند.

$$
\text { تشكر وقدردانى }
$$

اين يروهش با حمايت مالى دانشكده تربيث بدنى دانشكاه

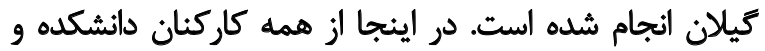
آزمودنى هاى تحقيق سياستزارى مئ دكنيه. 


\section{References}

[1] Hale SA, Hertel J. Reliability and sensitivity of the foot and ankle disability index in subject with chronic ankle instability. Journal of Athletic Training. 2005; 40(1):35-40. PMCID: PMC1088343

[2] Sefton JM, Hicks-Little CA, Hubbard TJ, Clemens MG, Yengo $\mathrm{CM}$, Koceja DM, et al. Sensorimotor function as a predictor of chronic ankle instability. Clinical Biomechanics. 2009; 24(5):4518. doi: 10.1016/j.clinbiomech.2009.03.003

[3] Shamway AC, Woollacott MH. Motor Control: Translating research into clinical practice. $4^{\text {th }}$ edition. Philadelphia: Lippincott Williams \& Wilkins; 2007.

[4] Khodabakhshi M, Ebrahimi-A'tri A, Hashemi Javaheri SA, KhanZadeh R, Zandi M. [The effect of 5 weeks proprioceptive training on basketball player's dynamic balance with aspirin for chronic ankle (Persian)]. Archives of Rehabilitation. 2014; 15(3):44-51.

[5] Dayakidis MK, Boudolos K. Ground reaction force data in functional ankle instability during two cutting movements. Clinical Biomechanics. 2006; 21(4):405-11. doi: 10.1016/j.clinbiomech.2005.11.010

[6] Pope M, Chinn L, Mullineaux D, McKeon PO, Drewes L, Hertel J. Spatial postural control alterations with chronic ankle instability. Gait \& Posture. 2011; 34(2):154-8. doi: 10.1016/j.gaitpost.2011.04.012

[7] Monaghan K, Delahunt E, Caulfield B. Ankle function during gait in patients with chronic ankle instability compared to controls. Clinical Biomechanics. 2006; 21(2):168-74. doi: 10.1016/j. clinbiomech.2005.09.004

[8] Hadadi M, Mazaheri M, Mousavi ME, Maroufi N, Bahramizadeh M, Fardipour S. Effects of soft and semi-rigid ankle orthoses on postural sway in people with and without functional ankle instability. Journal of Science and Medicine in Sport. 2011; 14(5):3705. doi: 10.1016/j.jsams.2010.12.004

[9] Richie DH. Effects of foot orthoses on patients with chronic ankle instability. Journal of the American Podiatric Medical Association. 2007; 97(1):19-30. doi: 10.7547/0970019

[10] Kennedy A, Hug F, Bilodeau M, Sveistrup H, Guével A. Neuromuscular fatigue induced by alternating isometric contractions of the ankle plantar and dorsiflexors. Journal of Electromyography and Kinesiology. 2011; 21(3):471-7. doi: 10.1016/j.jelekin.2011.02.001

[11] Paiehdar S, Saeedi H, Ahmadi A, Kamali M, Mohammadi M. [The comparison of the immediate effect of 3 functional, ucbl and modified ucbl foot orthotics im-pact on dynamic balance in subjects with flexible flatfoot (Persian)]. Archives of Rehabilitation. 2013; 14(4):66-73.

[12] Kazemi K, Abdollahi I, Arab AM. Evaluation of the electromyographic activity of distal and proximal muscles of the lower extremity after ankle sprain (Review article). Physical Treaments. 2013; 3(3):46-52.

[13] Fatahi M, Ali Ghasemi G, Mongashti Joni Y, Zolaktaf V, Fatahi F. The effect of lower extremity muscle fatigue on dynamic postural control analyzed by electromyography. Physical Treaments. 2016; 6(1):37-50.
[14] Gribble PA, Hertel J, Denegar CR, Buckley WE. The effect of fatigue and chronic ankle instability on dynamic postural control. Journal of Athletic Training.. 2004; 39(4):321-9. PMCID: PMC535524

[15] Haji-Maghsoudi M, Naseri N, Nouri-Zadeh S, Jalayi S. [Evidence of reliability for persian version of the "Cumberland Ankle Instability Tool (CAIT)" in Iranian athletes with lateral ankle sprain (Persian)]. Archives of Rehabilitation. 2016; 16(4):304-10.

[16] Perrin DH. Athletic taping and bracing. $2^{\text {nd }}$ edition. Champaign: Human Kinetics Books; 2005.

[17] Huang CY, Hsieh TH, Lu SC, Su FC. Effect of the kinesio tape to muscle activity and vertical jump performance in healthy inactive people. BioMedical Engineering OnLine. 2011; 10(1):70. doi: $10.1186 / 1475-925 x-10-70$

[18] Quackenbush KE, Barker PRJ, Stone Fury SM, Behm DG. The effects of two adhesive ankle-taping methods on strength, power, and range of motion in female athletes. Journal of Sports Physical Therapy. 2008; 3(1): 25-32. PMCID: PMC2953307

[19] Bahr R, Engebretsen L. Sport injury prevention. $1^{\text {st }}$ edition. New Jersey: Wiley-Blackwell; 2009.

[20] Shaw MY, Gribble PA, Frye JL. Ankle bracing, fatigue, and time to stabilization in collegiate volleyball athletes. Journal of Athletic Training. 2008; 43(2):164-71. doi: 10.4085/1062-6050-43.2.164

[21] Wikstrom EA, Arrigenna MA, Tillman MD, Borsa PA. Dynamic postural stability in subjects with braced, functionally unstable ankle. Journal of Athletic Training. 2006; 41(3):245-50. PMCID PMC1569562

[22] Ross SE, Guskiewicz KM, Yu B. Single- leg jump-landing stabilization times in subjects with functionally unstable ankle. Journal of Athletic Training. 2005; 40(4):298-304. PMCID: PMC1323291

[23] Wikstrom EA, Powers ME, Tillman MD. Dynamic stabilization time after isokinetic and functional fatigue. Journal of Athletic Training. 2004; 39(3):247-53. PMCID: PMC522147

[24] Delahunt E, McGrath A, Doran N, Coughlan GF. Effect of taping on actual and perceived dynamic postural stability in persons with chronic ankle instability. Archives of Physical Medicine and Rehabilitation. 2010; 91(9):1383-9. doi: 10.1016/j. apmr.2010.06.023

[25] Lohkamp M, Craven S, Walker-Johnson C, Greig M. The influence of ankle taping on changes in postural stability during soccer-specific activity. Journal of Sport Rehabilitation. 2009; 18(4):482-92. doi: $10.1123 /$ jsr.18.4.482

[26] Harkins KM, Mattacola CG, Uhl TL, Malone TR, McCrory JL. Effects of 2 ankle fatigue models on the duration of postural stability dysfunction. Journal of Athletic Training. 2005; 40(3):19194. PMCID: PMC1250260

[27] Bisson EJ, McEwen D, Lajoie Y, Bilodeau M. Effects of ankle and hip muscle fatigue on postural sway and attentional demands during unipedal stance. Gait \& Posture. 2011; 33(1):83-7. doi: 10.1016/j.gaitpost.2010.10.001

[28] Gribble PA, Taylor BL, Shinohara J. Bracing does not improve dynamic stability in chronic ankle instability subjects. Physical Therapy in Sport. 2010; 11(1):3-7. doi: 10.1016/j.ptsp.2009.11.003 
[29] Eechaute C, Vaes P, Van Aerschot L, Asman S, Duquet W. The clinimetric qualities of patient-assessed instruments for measuring chronic ankle instability: A systematic review. BMC Musculoskeletal Disorders. 2007; 8:6. doi: 10.1186/1471-2474-8-6

[30] Brown C, Ross S, Mynark R, Guskiewicz K. Assessing functional ankle instability with joint position sense, time to stabilization, and electromyography. Journal of Sport Rehabilitation. 2004; 13(2):122-34. doi: 10.1123/jsr.13.2.122

[31] Aune AK, Nordsletten L, Skjeldal S, Madsen JE, Ekeland A. Hamstrings and gastrocnemius co-contraction protects the anterior cruciate ligament against failure: Anin vivo study in the rat. Journal of Orthopaedic Research. 1995; 13(1):147-50. doi: 10.1002/jor.1100130122

[32] Myers JB, Guskiewicz KM, Schneider RA, Prentice WE. Proprioception and neuromuscular control of the shoulder after muscle fatigue. Journal of Athletic Training. 1999; 34(4):362-7. PMID: 16558590

[33] Shills JJ, Kaminski TW, Tillman MD. Comparing time to stabilization values following an acute bout of intensive exercise in those with functional ankle instability. Journal of Athletic Training. $2003 ; 38(2): 50$.

[34] Forestier N, Teasdale N, Nougier V. Alteration of the position sense at the ankle induced by muscular fatigue in humans. Medicine \& Science in Sports \& Exercise. 2002; 34(1):117-22. doi: 10.1097/00005768-200201000-00018

[35] Zhang SN, Bates BT, Dufek JS. Contributions of lower extremity joints to energy dissipation during landings. Medicine \& Science in Sports \& Exercise. 2000; 32(4):812-9. doi: 10.1097/00005768200004000-00014

[36] Iris M, Monterde S, Salvador M, Salvat I, Fernandez-Ballart J, Judith B. Ankle taping can improve proprioception in healthy volunteers. Foot \& Ankle International. 2010; 31(12):1099-106. doi: 10.3113/fai.2010.1099

[37] Pedowitz DI, Reddy S, Parekh SG, Huffman GR, Sennett BJ. Prophylactic bracing decreases ankle injuries in collegiate female volleyball players. American Journal of Sports Medicine. 2008; 36(2):324-7. doi: 10.1177/0363546507308358

[38] Gribble PA, Robinson RH. Alterations in knee kinematics and dynamic stability associated with chronic ankle instability. Journal of Athletic Training. 2009; 44(4):350-5. doi: 10.4085/1062-605044.4.350

[39] Ozer D, Senbursa G, Baltaci G, Hayran M. The effect on neuromuscular stability, performance, multi-joint coordination and proprioception of barefoot, taping or preventative bracing. Foot. 2009; 19(4):205-10. doi: 10.1016/j.foot.2009.08.002

[40] Hopper D, Samsson K, Hulenik T, Ng C, Hall T, Robinson $\mathrm{K}$. The influence of Mulligan ankle taping during balance performance in subjects with unilateral chronic ankle instability. Physical Therapy in Sport. 2009; 10(4):125-30. doi: 10.1016/j. ptsp.2009.07.005

[41] Somehe M, Norasteh AA, Daneshmandi H, Pourkhani T. [The influence of Mulligan ankle taping on dynamic balance in the athletes with and without chronic ankle instability (Persian)]. Archives of Rehabilitation. 2014; 15(1):70-77.
[42] Olmsted L, Vela L, Denegar CR, Hertel J. Prophylactic ankle taping and bracing: A numbers-needed-to treat and cost benefit analysis analysis. Journal of Athletic Training. 2004; 39(1):95-100. PMCID: PMC385268 